\title{
PHILOElocite
}

Philologica Canariensia 23 (2017), pp. 79-89 eISSN: 2386-8635

DOI: 10.20420/PhilCan.2017.150

Recibido: 10 de febrero de 2017; versión revisada aceptada: 25 de abril de 2017

Publicado: 26 de junio de 2017

\section{LA RECOLECCIÓN DE NARRACIONES ORALES Y SU ESTUDIO EN CORRELACIÓN CON FACTORES SOCIALES: EL VALOR DE LA ENTREVISTA SOCIOLINGÜÍSTICA*}

\author{
SILVANA GUERRERO GONZÁLEZ \\ Universidad de Chile
}

RESUMEN: Este trabajo reflexiona sobre la entrevista sociolingüística como técnica de recolección de narraciones de experiencia personal para el análisis sociolingüístico. Se intenta responder a las principales críticas que ha recibido esta técnica de recolección de datos y se ofrecen casos concretos que corroboran su importancia. Los datos presentados permiten comprobar que los hallazgos sobre narraciones no dependen del escenario en el que estas se generen. En consecuencia, la propuesta de estructura narrativa de Labov y Waletzky (1967) y de Labov (1972) es la que se utiliza, en general, en la construcción de cualquier narración de experiencia personal.

PALABRAS CLAVE: sociolingüística variacionista, variación discursiva, narraciones de experiencia personal, narraciones conversacionales, entrevista sociolingüística

The collection of oral narratives and their study in correlation with social factors: the value of the sociolinguistic interview

\begin{abstract}
This article examines the sociolinguistic interview as a technique for collecting personal experience narratives for sociolinguistic analysis. My aim is to try to answer the main critiques that this data collection technique has received and to present concrete cases that corroborate its importance. The data provided allows to prove that the findings concerning these narratives do not depend on the scenario in which they are generated. This is why Labov and Waletzky's (1967) and Labov's (1972) narrative structure is generally used for the construction of any personal experience narrative.

KEYWORDS: variationist sociolinguistics, discursive variation, personal experience narratives, conversational narratives, sociolinguistic interview
\end{abstract}

\section{INTRODUCCIÓN}

En este trabajo presentamos una reflexión acerca de la técnica que ha sido utilizada con mayor frecuencia para recolectar datos narrativos útiles en los estudios sociolingüísticos. Se comienza exponiendo una breve síntesis acerca de lo que entendemos por "narración” y luego ofrecemos algunos datos que permiten corroborar la relevancia de la entrevista sociolingüística para la confección de corpus de narraciones de experiencia personal (Labov, 1972), consideradas como un tipo de relato fundamental para los estudios sociolingüísticos, tal y como señala Labov $(2013,5)$, para quien la narración misma se ha convertido en "foco de interés lingüístico". Finalmente, discutimos sobre las principales críticas que ha recibido la entrevista semidirigida como medio de obtención de discurso vernáculo.

Este artículo se distribuye bajo una licencia Creative Commons Reconocimiento-NoComercial-SinObraDerivada 4.0 


\section{2. ¿NARRACIÓN, HISTORIA O RELATO? CARACTERÍSTICAS DEL OBJETO DE ESTUDIO EN SOCIOLINGÜÍSTICA VARIACIONISTA}

Suele hablarse indistintamente de narración, historia y relato. Por consiguiente, es relevante definir el que ha sido el tipo de discurso que ha servido de base para un sinnúmero de investigaciones en sociolingüística. Charaudeau y Maingueneau (2005) señalan que la narración es el acto de contar. El relato, en cambio, es una representación de una sucesión temporal de acciones y debe haber una transformación más o menos importante de ciertas propiedades iniciales de los actantes, así como una puesta en intriga que estructure y otorgue sentido a esa sucesión de acciones y acontecimientos en el tiempo. Siguiendo a Genette (1972, 1983), ambos autores plantean que el relato es el estrato verbal que se hace cargo de poner la historia en el texto.

Para Riessman (2008), la narración es sinónimo de historia. Todos los días narramos historias; el que habla conecta eventos dentro de una secuencia y les da un significado. Los eventos percibidos por el hablante como importantes son seleccionados, organizados, conectados y evaluados con un significado para una audiencia particular. Norrick (2000), por su parte, pone de relieve que la historia, la narración y el relato se usan en el sentido habitual en que se generan en la vida cotidiana. Se trata de una interacción cara a cara, dinámica, que cambia frecuentemente de orador. Lo importante es, para este autor, que la narración crece y se desarrolla en el contexto de una conversación concreta.

Lo que se desprende de lo anterior es que tanto narración como relato e historia podrían aplicarse al estudio sociolingüístico de la narrativa oral. Ya que los tres términos funcionan de manera indistinta, la única precisión que haremos al respecto es que en este trabajo nos centramos en las narraciones o relatos de experiencia personal que deben ser dignos de contarse, esto es, ser reportables. Según Labov (2004), cualquier relato dado se construye alrededor de un evento más reportable. Sin embargo, en el proceso de narrar se produce una relación inversa entre informar sobre los hechos ocurridos y la credibilidad que estos tengan, pues que un evento sea contable lo hace también poco común, raro, poco probable y, por lo tanto, menos creíble (Labov, 1997), asunto que se soluciona en la medida en que se trate de narraciones sobre hechos que se supone ocurrieron realmente (Labov, 1972, 360 y ss.; Labov, 2013, 21). De acuerdo con la propuesta laboviana, las narraciones cuentan, además, con una linealidad temporal o causalidad, un punto de vista, hechos objetivos y hechos subjetivos (Labov, 1997). Todas las características mencionadas son esenciales en el momento de llevar a cabo el análisis de las narraciones desde la perspectiva sociolingüística, porque son estas las que configuran la estructura fundamental de un relato de experiencia personal.

\section{LA NARRACIÓN ORAL DE EXPERIENCIA PERSONAL Y LOS APORTES ESTRUCTURALES DE WILLIAM LABOV}

Dos de las tesis más comunes en la bibliografía sobre narraciones son, por una parte, que la narración oral es una de las estructuras textuales más interesantes y complejas que pueden suscitarse dentro de las conversaciones y, por otra, que los sujetos necesitan que sus vidas 
sean contables e interesantes para crear identidades individuales (Moreno Fernández, 1998; Özyildirim, 2009). Las historias orales se caracterizan por ser incompletas y selectivas e, independientemente de que lo que el narrador cuente sea o no autobiográfico, este siempre representa su papel desde una posición ventajosa, pues construye el mundo de la historia de acuerdo con sus propias referencias espacio-temporales, selecciona las acciones y los personajes que las desarrollan y elige la perspectiva desde la cual verbalizar su narración (Camargo, 2004, 2006, 2010). Labov (2001, 2004) señala que las narraciones de experiencia personal adquieren, en especial, un papel destacado en los estudios sociolingüísticos porque en la entrevista sociolingüística conforman aquella sección en la que se puede obtener un discurso vernáculo, ${ }^{1}$ que es el que sociolingüísticamente conviene analizar (Labov, 2004).

Labov (1997, 396 y ss.) destaca el trabajo de Labov y Waletzky (1967) donde se demuestra que las narraciones son susceptibles de un marco formal, en particular en la definición básica de la narración como la elección de una técnica lingüística específica para informar sobre hechos ocurridos en el pasado. Los presupuestos teóricos de estos autores han demostrado ser útiles para abordar una amplia variedad de situaciones narrativas. En todos estos casos, lo que se comprueba es que las narraciones son formas privilegiadas de discurso que desempeñan un papel central en casi todas las conversaciones y que, además, en su versión prototípica, son un ejemplo de evento de habla bien formado, es decir, con un principio, un medio y un fin (Labov, 1997).

Estrictamente hablando, Labov $(1972,360)$ define la narración como "one method of recapitulating past experience by matching a verbal sequence of clauses to the sequence of events which (it is inferred) actually occurred”. Labov y Waletzky (1967) y Labov (1972) distinguen entre relatos mínimos y relatos completos. Los primeros están compuestos de, al menos, dos cláusulas narrativas en pasado, mientras que los segundos exhiben seis rasgos estructurales claramente definidos: resumen, orientación, acción de complicación, evaluación, resolución, y coda. ${ }^{2}$

Tómese como ejemplo de un relato completo el que sigue a continuación, extraído del corpus del Proyecto para el Estudio Sociolingüístico del Español de España y de América (PRESEEA) de Santiago de Chile. Se trata, asimismo, de una narración bien constituida, puesto que en ella los acontecimientos se disponen en orden lógico-temporal, característica esencial del relato en la teoría laboviana. Para mayor claridad en la lectura, distinguimos cada parte de la estructura narrativa con un sistema de etiquetas de inicio $<>$ y de cierre $</>$ y subrayaremos los elementos evaluativos, transversales en el relato:

I: <resumen> cuando casi me asaltaron </resumen > fue esa vez que / que ahí <orientación>en calle / en Bombero Ossa en el centro venía / estaba haciéndole un cambio a mis hermanos un cambio en dólares / pero esa vez yo tenía que cambiar mi / la plata eeh chil[ena] en dólares a chilena y llevaba como siete millones de pesos en mi bolsillo </orientación $>$ / <complicación de la narración $>$ y un y un tipo me quiso contar el cuento del tío / entonces yo le creí / que andaba buscando una dirección y me llevó a un a una galería / apareció una niña que me decía que lo ayudara // y y lo llevé a la galería Alessandri y lo llevé al segundo piso / porque él insistía que en el segundo piso parece que era / y cuando llego al segundo piso / me doy cuenta de que habían puras oficinas cerradas / y como nos demoramos un poco / por otra por una puerta / por otra / por otro 
acceso que había en el en el segundo piso aparece la misma mujer que estaba abajo // entonces yo saqué por conclusión que me querían asaltar </complicación de la narración $>$ / <resolución $>\mathrm{y}$ arranqué / corriendo por las escalas hacia abajo y ahí yo me di cuenta porque la mujer empezó a retar al hombre que se había demorado mucho en llevarme a un a una / a un pasillo que no tenía salida y si no habría </resolución > //E: ¿era viejito? //I: sí era más o menos entre unos cincuenta sesenta años // y y ¿cómo es que se llama? y si me hubiese metido a ese pasillo me hubiesen robado toda la plata que eran como siete millones de pesos //E: qué horrible// I: sí // <coda>ese también fue un susto grande $</ \operatorname{coda}>$ (SCHI_M22_057 PRESEEA-SA).

En el ejemplo precedente, se puede observar cómo la historia se va construyendo de manera que quedan claros los eventos que conforman el principio, el medio y el fin de los hechos. Se presenta la secuencia de eventos del pasado y se utilizan múltiples recursos de evaluación -como discurso referido y presente histórico- para mostrar el punto de vista del narrador.

\subsection{La entrevista sociolingüística}

En ciencias sociales la entrevista es una de las técnicas de campo más utilizadas. Sin embargo, la elección del instrumento se vincula directamente con el tipo de material que el investigador desea obtener y, como es lógico, con su objeto de estudio. En este orden, la entrevista semidirigida (o semiestructurada) se constituye como uno de los medios más eficaces para obtener corpus de análisis sociolingüísticos. Moreno Fernández (2012, 179) indica que la entrevista sociolingüística es la técnica que se ha "manejado como la principal fuente para la provisión de materiales analizables desde una perspectiva cuantitativa variacionista". Según este autor, "la entrevista semidirigida es el medio más adecuado para obtener muestras de lengua hablada en la cantidad y con la calidad necesarias para su análisis cuantitativo" (Guerrero, 2014b, 96). Asimismo, la entrevista semidirigida es el medio óptimo para registrar sistemáticamente el discurso vernáculo, lo que implica, en consecuencia, no olvidar el efecto de la denominada paradoja del observador. Para Labov (1983 [1972]), el objetivo de la investigación lingüística de una comunidad de habla consiste en estudiar la forma en que habla la gente cuando no está siendo sistemáticamente observada; sin embargo, es solo mediante la observación sistemática como podemos obtener tales datos. A esto es a lo que se le denomina la paradoja del observador. Este dilema se soluciona encontrando la manera de completar la entrevista formal con otros datos, o cambiando de algún modo la estructura de la situación de la entrevista. En el marco de esta, se debe generar un clima de confianza, de manera que los hablantes olviden que están siendo grabados.

La entrevista laboviana consta de varias secciones, 3 cada una de las cuales está destinada a obtener distintos tipos de discurso y diversos estilos, desde el más formal al más vernáculo. La sección que es pertinente para nuestro trabajo es la de la "narración de experiencias personales". Las preguntas de esta sección están destinadas a obtener relatos de experiencias significativas para el informante, como anécdotas, sustos, accidentes, enfermedades, vergüenzas y recuerdos, en general. La bibliografía acerca del tema señala que aquí el informante se siente directa e íntimamente involucrado en su relato, de manera tal que se 
relaja y pierde la noción de entrevista en la que se halla (cf. Hernández Campoy y Almeida, 2005, 136). 4

\subsection{Las críticas y los aportes de los estudios sociolingüísticos sobre la narración oral}

En lo que respecta al enfoque sociolingüístico, las investigaciones sobre narrativas han construido las muestras de estudio sobre la base de relatos que se producen en el marco de una entrevista sociolingüística, donde el entrevistador constituye un público ideal, pues es, según Labov (1997), atento, interesado y receptivo. No obstante, son muchas las críticas que ha recibido este enfoque. Norrick (2000) y Juzwik (2012) señalan que Labov (1972) se concentra en narrativas completas, orales, de experiencia personal desde la perspectiva del entrevistado, pero no considera los efectos de la interacción. También Thornborrow (2012) destaca que la narración es un fenómeno interactivo y que Labov no solo no trata el discurso narrativo contextualmente situado, sino que, además, no presta atención a aspectos de la interacción. El mismo énfasis es otorgado por De Fina y Johnstone (2015), quienes precisan que Labov solo se concentra en la producción de cláusulas del narrador y no considera las contribuciones de los demás participantes o de la audiencia general, ni tampoco el contexto interaccional. En cierta medida, en la entrevista la situación comunicativa no es natural y se trata esencialmente de monólogos. Las críticas apuntan a que en la conversación espontánea los relatos son a menudo muy fragmentarios y pueden requerir un enfoque de análisis diferente.

Sin embargo, señala Labov (1997), los estudios de la conversación espontánea también muestran una alta frecuencia de narraciones monológicas, donde se demanda la atención de la audiencia tanto como en los relatos de la entrevista sociolingüística. Con base en este supuesto, son muchas las investigaciones que han abordado el estudio de las narraciones de experiencia personal siguiendo la propuesta de la estructura narrativa laboviana. Un conjunto importante de estudios se ha centrado en mostrar cómo se relacionan los relatos con la construcción y la expresión del sexo-género de los hablantes (cf. Silberstein, 1988; Johnstone, 1990; Tannen, 1996; Reyes, 2002; Soler, 2004; Coates, 2009). Otro aspecto importante es el que se vincula con el análisis de la oralidad (cf. Prieto y San Martín, 20022003; Camargo, 2004; Gallucci, 2012; San Martín y Guerrero, 2013; Guerrero, 2014a, 2014c). En relación con el factor edad y la narración, la bibliografía es escasa. Los trabajos provienen en general de comunidades de habla inglesa (cf. Labov, 1972). La relación entre la edad y la producción de narraciones ha sido ampliamente estudiada en el campo de la cognición y de la psicolingüística. En lo que respecta al estudio variacionista de la narración en correlación con el factor edad, destacamos los trabajos en el español hablado en Santiago de Chile vinculados con el grupo etario juvenil de Guerrero (2011a, 2011b). La clase social y su incidencia en la elaboración de narraciones de experiencia personal también ha sido poco investigada. Los estudios se concentran, sobre todo, en el ámbito angloparlante (cf. Labov y Waletzky, 1967; Horvath, 1987; Macaulay, 1991, citado en Smith, 2005; Reyes, 1996).5

En otro orden, Johnstone (1990, 2001, 2006) señala que Labov y Waletzky (1967) presentan un estudio de la sintaxis narrativa en el contexto de la investigación 
sociolingüística variacionista, pero en la mayor parte de los trabajos la narrativa no es el objeto de análisis. Su argumento es que los estudios existentes no se han detenido a analizar la manera en que la estructura y las funciones propias de la narrativa podrían influir en la variación y el cambio lingüístico. Lo que esta autora propone es que la estructura y la función de la narrativa, en cuanto proceso de construcción y narración resultante, pueden arrojar luz sobre la formación de la lengua vernácula, ya que podrían existir conjuntos particulares de rasgos lingüísticos vinculados con la región, la clase y otras fuentes de identidad, incluidas aquellas relacionadas con el lenguaje ideológico. Esto también se sugiere en los trabajos de Guerrero (2013, 2015).

\section{RESPONDIENDO A LAS CRÍTICAS}

Atendiendo a algunas de las principales críticas que ha recibido la entrevista sociolingüística como técnica de recolección de datos para el estudio de la narración oral, Guerrero (en prensa) compara la estructuración de narraciones de experiencia personal producidas individualmente por hablantes santiaguinos (Prieto, 1995-1996) del grupo de edad que va entre los 35 y los 54 años con narraciones conversacionales generadas por hablantes de similares características. Las narraciones fueron extraídas de dos corpus: a) 36 narraciones pertenecientes al corpus sociolingüístico del Proyecto para el Estudio Sociolingüístico del Español de España y de América correspondiente a la variedad de Santiago de Chile (PRESEEA-SA), las cuales fueron construidas de manera individual y obtenidas mediante entrevista sociolingüística, y b) 54 narraciones que forman parte del proyecto "Entre la sociolingüística variacionista y la sociolingüística interaccional: un análisis génerolectal y sociolectal de la co-construcción de narraciones de experiencia personal”. Este grupo de narraciones son co-construidas (conversacionales o interaccionales), grabadas audiovisualmente, y obtenidas a través del diseño narrativo para el estudio autobiográfico y de tópico. ${ }^{6}$

El análisis contrastivo de los datos proporcionados por Guerrero permite verificar la aplicabilidad de la propuesta estructural de la narración desarrollada por Labov y Waletzky (1967) y por Labov (1972) no solo a relatos registrados en un escenario ideal y no competitivo, sino también a aquellos que se generan en el marco de la interacción. Los resultados muestran cifras prácticamente idénticas, esto es, el resumen se presenta en el $44,4 \%$ de las narraciones obtenidas en entrevistas sociolingüísticas, en tanto que en las narraciones conversacionales aparece en el 53,7\% de los casos. La orientación se incluye en el 91,6\% de las narraciones individuales y en un 98,1\% de las conversacionales. La complicación del relato se observó en el $86,1 \%$ de las historias de entrevistas y en el $87 \%$ de las historias interaccionales. Por su parte, la resolución se incluye en el 52,7\% de las narraciones individuales y en el 70,4\% de las conversacionales. Finalmente, la coda aparece en el 66,6\% de las narraciones de entrevista y en el 72,2\% de las historias co-construidas. La evaluación se presenta en el 100\% de los relatos analizados (cf. Guerrero, en prensa). En consecuencia, que la narrativa se genere o no en una entrevista sociolingüística no parece ser un factor determinante en su construcción. 
La bibliografía señala que entre los rasgos de la entrevista sociolingüística destaca su poco o nulo dinamismo y su poca espontaneidad; por lo tanto, una de las misiones del entrevistador es atenuar la rigidez de la situación, cuyos rasgos implican, entre otros, la disposición de los participantes, la estructura del discurso y su contenido (Moreno Fernández, 2012). ${ }^{7}$ A medida que se supera la rigidez de la situación de entrevista, los resultados han demostrado ser aptos para la investigación sociolingüística; consecuentemente, los presupuestos labovianos sobre la narración de experiencia personal serían también aplicables a los relatos que se generan en la conversación coloquial. Parece ser que, tal y como postula Labov (1972), existe una estructura semántica profunda invariante de los relatos que se relaciona con las diferencias superficiales ocasionadas por las características sociales de los hablantes. Siguiendo los planteamientos de De Fina y Johnstone (2015), lo relevante en las narraciones interaccionales es que los participantes influyen en la narración como co-narradores, pero esto no afecta el desarrollo estructural del relato.

Según Guerrero (en prensa), el análisis referido a la sensibilidad de las narraciones a la variación en correlación con los factores sexo y grupo socioeconómico también permite verificar, en términos descriptivos, que pese a la relevancia que tiene estudiar el relato en su contexto interaccional, la propuesta estructural de Labov para la narración es muy similar entre narraciones "ideales" y aquellas que se generan por más de un narrador. La naturaleza interactiva de la narración no se contradice con los presupuestos de Labov acerca de la narración prototípica como evento de habla bien formado, con principio, medio y fin, y con constituyentes básicos.

\section{REFLEXIONES FINALES}

En este breve trabajo hemos expuesto nuestra reflexión sobre las técnicas que han sido utilizadas con mayor frecuencia para recolectar datos narrativos útiles en los estudios cuyo enfoque es primordialmente el de la sociolingüística variacionista. Los datos presentados verifican la relevancia de la entrevista sociolingüística para la confección de corpus de narraciones de experiencia personal (Labov 1972) y permiten comprobar que la propuesta de estructura narrativa laboviana es la que se utiliza, en general, en la construcción de cualquier forma de narrativa, sin que ello implique que no existan diferencias a nivel de superficie en lo narrado, como rasgos propios de la orientación o de la evaluación narrativas.

\section{NOTAS}

* Este trabajo se enmarca en el Proyecto FONDECYT 11150007, cuyo propósito es estudiar la co-construcción de narraciones de experiencia personal en relación con los factores génerolectal y sociolectal en una muestra de hablantes de Santiago de Chile. La redacción final se realizó en el contexto de una pasantía de investigación en el Departamento de Filología, Comunicación y Documentación de la Universidad de Alcalá (Madrid), en el marco del Proyecto de Estímulo de la Excelencia Institucional (PEEI) impulsado y financiado por las Vicerrectorías de Asuntos Académicos y de Investigación y Desarrollo de la Universidad de Chile. 
1 Moreno Fernández (2012, 181-182), citando a Labov, señala que "[e]l vernáculo, en el que se presta al discurso propio una atención mínima, aporta los datos más sistemáticos para el análisis lingüístico. El vernáculo se define como la forma de hablar adquirida durante la preadolescencia”.

2 Autores como Moreno Fernández (2009) se refieren al resumen como inserción. La coda y el resultado (o resolución) tienen funciones que coinciden parcialmente. La primera señala explícitamente que la historia ha terminado, a través de expresiones como "Y eso fue todo", "Y eso fue lo que nos contó", "Y así terminó la cosa", ejemplos proporcionados por Silva-Corvalán (2001). Por su parte, el resultado señala lo que aconteció finalmente.

3 En la práctica, cada equipo de investigación ha modificado el cuestionario a fin de obtener los datos pertinentes para el tipo de investigación que desea desarrollar, aunque la estructura básica de la entrevista laboviana ha tendido a mantenerse.

$4 \quad$ El cuestionario para la entrevista sociolingüística utilizado por el equipo del PRESEEA de Santiago de Chile incluye preguntas como: “¿Recuerda(s) alguna anécdota graciosa/jocosa de su/tu época de estudiante, ya sea de enseñanza básica, enseñanza media o universitaria?”, “¿Cuál ha sido el susto más grande que le/te ha tocado pasar?”, “¿Ha(s) estado alguna vez en una situación tal que su/tu salud o su/tu vida hayan estado en serio riesgo?”, “¿Cómo vivió/viviste el terremoto del 27 de febrero de 2010?”, “¿Cuál ha sido la vergüenza/plancha más grande que le/te ha tocado pasar?”, “¿Cuál ha sido el recuerdo más grato de su/tu vida, es decir, que le haya quedado grabado en la memoria?”, “¿Cuáles han sido las mejores vacaciones que le/te han tocado pasar? ¿Qué hizo de esas vacaciones algo tan inolvidable?”, entre otras.

5 Aun cuando no es nuestro propósito sintetizar detalladamente los hallazgos acerca de investigaciones sobre narraciones orales en sociolingüística, presentamos algunos resultados relevantes. Johnstone (1990), concluye que los hombres cuentan relatos, entre otras cosas, de pruebas en las que se ven enfrentados a otros hombres, de hazañas que exaltan sus habilidades, su coraje o su ingenio, y casi nunca incluyen a mujeres como protagonistas de sus narraciones. Silberstein (1988) señala que los relatos de mujeres se centran en la toma de decisiones y en cómo justificarlas ante los hombres, mientras que ellos hablan sobre planes y conquistas. Coates (2009), por su parte, concluye que contar historias es esencial en todo grupo de amigas y que los temas versan, en general, sobre rutinas de la vida diaria (labores domésticas, hijos o esposo). Asimismo, Soler (2004) apunta, entre otras cosas, que, respecto a la estructura de los relatos, los de las mujeres tienen prácticamente el doble de extensión que los de los hombres y que, pese a que en ambos sexos se observan las seis partes de la narración laboviana, la distribución y función de las mismas varía en hablantes de distinto sexo. En cuanto a la edad, Labov (1972) propone que, a medida que aumenta la edad, también aumenta la habilidad de los hablantes para construir narraciones. Así, desde el campo de la ciencia cognitiva se ha determinado que desde los cuatro años los niños pueden formular relatos, pero de manera poco sistemática. Por último, sobre el factor grupo social, Labov y Waletzky (1967), por ejemplo, observan diferencias entre clases sociales, a saber, que los hablantes de la clase media alta reportan emociones, mientras que los de la clase baja incorporan pocos elementos subjetivos en sus relatos. Horvath (1987), en otro orden, destaca que los informantes de la clase trabajadora hablan en sus historias de ellos mismos y de otros miembros de sus familias, en tanto que los hablantes de clase media pueden incluir personajes a los que no conocen directamente.

6 El diseño narrativo permite recopilar datos sobre historias de vida y experiencias personales para su descripción y análisis (Riessman, 2008). Mertens (2005) divide en tres los estudios narrativos: a) de tópico, b) biográfico y c) autobiográficos. Estos estudios apuntan a la descripción de la construcción del yo y de la identidad a través de la expresión de narrativas. 
7 En ninguno de los protocolos disponibles para la grabación de entrevistas sociolingüísticas se señala que el entrevistador ha de atenuar esta rigidez. Se trata, en consecuencia, de una tarea pendiente.

\section{REFERENCIAS BIBLIOGRÁFICAS}

CAMARGO FERNÁNDEZ, L. 2004. La representación del discurso en la narración oral conversacional. Estudio sociopragmático, Tesis doctoral. Madrid: Universidad de Alcalá.

CAMARGO FERNÁNDEZ, L. 2006. "El diálogo reconstruido y la imagen positiva”, en Casado, M., González, R., y Romero-Gualda, M. V. (dirs.), Análisis del discurso: lengua, cultura, valores. Vol. I. Madrid: Arco/Libros, 1241-1253.

CAMARGO FERNÁNDEZ, L. 2010. "Dialogues Within Oral Narratives: Functions and Forms", en Koike, D. A. y Rodríguez-Alfano, L. (eds.), Dialogue in Spanish. Studies in Functions and Contexts. Amsterdam/Filadelfia: John Benjamins, 31-54.

CHARAUDEAU, P. y MAINGUENEAU, D. 2005. Diccionario de análisis del discurso. Buenos Aires: Amorrortu.

COATES, J. 2009. Mujeres, hombres y lenguaje. Un acercamiento sociolingüístico a las diferencias de género. México: Fondo de Cultura Económica.

DE FINA, A. y JOHNSTONE, B. 2015. "Discourse Analysis and Narrative", en Tannen, D., Hamilton, H. E., y Schiffrin, D. (eds.), The Handbook of Discourse Analysis. Malden, Mass.: Wiley Blackwell, 152-168.

GALLUCCI, M. J. 2012. "Estilo directo e indirecto en interacciones orales. Estado de la cuestión en el ámbito hispánico", Boletín de Filología, XLVII(2), 205-233.

GENETTE, G. 1972. Figures III. París: Seuil.

GENETTE, G. 1983. Nouveau discours du récit. París: Seuil.

GUERRERO, S. 2011a. "Análisis sociolingüístico de las diferencias de género en patrones narrativos de historia de experiencia personal en el habla juvenil de Santiago de Chile”, Boletín de Filología, XLVI(2), 85-106.

GUERRERO, S. 2011b. "Diferencias de género en evaluaciones de narraciones de experiencia personales en el habla juvenil de Santiago de Chile. Una aproximación sociolingüística”, Revista Signos, 44(75), 18-32.

GUERRERO, S. 2013. "Sobre la aplicación de la perspectiva sociolingüística al estudio de la variación discursiva: el caso de la narración de experiencia personal”, Onomázein, 28, 188-200.

GUERRERO, S. 2014a. "Un análisis variacionista del discurso referido en el español de Santiago de Chile”, Signos lingüísticos, 10(20), 46-77.

GUERRERO, S. 2014b. "Una aproximación sociolingüística al empleo del presente histórico en narraciones de experiencia personal en hablantes de Santiago de Chile". RLA. Revista de Lingüística Teórica y Aplicada, 52(2), 89-112.

GUERRERO, S. 2014c. Variación discursiva en narraciones de experiencia personal en el español hablado en Santiago de Chile. Tesis doctoral. Chile: Pontificia Universidad Católica de Chile.

GUERRERO, S. 2015. "Una propuesta de clasificación de los estilos discursivos en narraciones de experiencia personal desde el punto de vista sociolingüístico”, Oralia, 18, 103-129. 
GUERRERO, S. (en prensa). "Narración individual versus narración conversacional: un puente entre el variacionismo y la interacción”, Onomázein, 38.

HERNÁNDEZ CAMPOY, J. M. y ALMEIDA, M. 2005. Metodología de la investigación sociolingüística. Málaga: Comares.

HORVARTH, B. 1979. "Text on Conversation: Variability in Storytelling Texts", en Denning, K. M., Inkelas, S, McNair-Knox, F., y Rickford, J. (eds.), Variation in Language: $N W A V-X V$ at Stanford. Stanford, CA: Department of Linguistics, Stanford University.

JOHNSTONE, B. 1990. "Variation in Discourse: Midwestern Narrative Style", American Speech, 65(3), 195-214.

JOHNSTONE, B. 2001. "Discourse Analysis and Narrative", en Schiffrin, D., Tannen, D., y Hamilton, H. E. (eds.), The Handbook of Discourse Analysis. Malden, Mass.: Blackwell, 635-649.

JOHNSTONE, B. 2006. “A New Role for Narrative in Variationist Sociolinguistics", Narrative Inquiry, 1, 46-55.

JUZWIK, M. 2012. "Spoken Narrative", en Gee, J. P. y Handford, M. (eds.), The Routledge Handbook of Discourse Analysis. Londres: Routledge, 326-341.

LABOV, W. 1972. "The Transformation of Experience in Narrative Syntax", en Language in the Inner City. Studies in the Black English Vernacular. Filadelfia: University of Pennsylvania Press, 354-396.

LABOV, W. 1983 [1972]. Modelos sociolingüísticos. Madrid: Cátedra.

LABOV, W. 1997. "Some Further Steps in Narrative Analysis", Journal of Narrative and Life History, 7, 395-415.

LABOV, W. 2001. "Uncovering the Event Structure of Narrative", en Tannen, D. y Alatis, J. E. (eds.), Linguistics, Language, and the Real World: Discourse and Beyond, Georgetown University Round Table on Languages and Linguistics. Washington: Georgetown University Press, 63-83.

LABOV, W. 2004. "Ordinary Events", en Fought, C. (ed.), Sociolinguistic Variation: Critical Reflections. Oxford: Oxford University Press, 31-43.

LABOV, W. 2006. "Narrative Pre-construction", Narrative Inquiry, 1, 37-45.

LABOV, W. 2013. The Language of Life and Death. The Transformation of Experience in Oral Narrative. Cambridge: Cambridge University Press.

LABOV, W. y WALETZKY, J. 1967. "Narrative Analysis", en Helm, J. (ed.), Essays on the Verbal and Visual Arts. Seattle: University of Washington Press, 12-44.

MERTENS, D. 2005. Research and Evaluation in Education and Psychology: Integrating Diversity with Quantitative, Qualitative, and Mixed Methods. Thousand Oaks: Sage.

MORENO FERNÁNDEZ, F. 1990. Metodología sociolingüística. Madrid: Gredos.

MORENO FERNÁNDEZ, F. 1998. Principios de sociolingüística y sociología del lenguaje. Barcelona: Ariel.

MORENO FERNÁNDEZ, F. 2012. Sociolingüística cognitiva. Proposiciones, escolios y debate. Madrid/Frankfurt: Iberoamericana/Vervuert.

NORRICK, N. R. 2000. Conversational Narrative: Storytelling in Everyday Talk. Amsterdam/ Philadelphia: John Benjamins. 
ÖZYILDIRIM, I. 2009. "Narrative Analysis: An Analysis of Oral and Written Strategies in Personal Experience Narratives", Journal of Pragmatics, 41(6), 1209-1222.

PRESEEA (2014- ). Corpus del Proyecto para el estudio sociolingüístico del español de España y de América. Alcalá de Henares: Universidad de Alcalá. Disponible en: http://preseea. linguas.net.

PRIETO VERA, L. 1995-1996. “Análisis sociolingüístico del dequeísmo en el habla de Santiago de Chile”, Boletín de Filología, XXXV, 379-452.

PRIETO VERA, L. y SAN MARTÍN, A. 2002-2003. "Diferencias de género en el empleo del discurso referido: aproximación sociolingüística y pragmático-discursiva”, Boletín de Fỉlología, XXXIX, 269-303.

REYES, C. 1996. "Estrategias narrativas en la zona metropolitana de Monterrey", en Rodríguez Flores, D. E. y Rodríguez Alfano, L. (eds.), Lenguaje y sociedad: metodología y análisis aplicados al habla de Monterrey. México: Trillas, 101-120.

REYES, C. 2002. "Algunas diferencias en las narraciones de hombres y mujeres dentro del corpus de 'El habla de Monterrey", Iztapalapa, 53, 101-115.

RIESSMAN, C. K. 2008. Narrative Methods for the Human Sciences. Thousand Oaks: Sage.

SAN MARTÍN, A. y GUERRERO, S. 2013. "Una aproximación sociolingüística al empleo del discurso referido en el corpus PRESEEA de Santiago de Chile”, Revista Signos, 46(82), 258-282.

SILBERSTEIN, S. 1988. "Ideology as Process: Gender Ideology in Courtship Narratives", en Todd, A. y Fisher, S. (eds.), Gender and Discourse: The Power of Talk. Nueva York: Norwood, 95-120.

SILVA-CORVALÁN, C. 2001. Sociolingüística y pragmática del español. Washington: Georgetown University Press.

SMITH, J. 2005. "Narrative: Sociolinguistics Research", en Brown, K., Asher, R. E., y Simpson, J. (eds.), The Encyclopedia of Language and Linguistics. Oxford: Pergamon, 289-309.

SOLER, S. 2004. Discurso y género en historias de vida. Una investigación de relatos de hombres y mujeres en Bogotá. Bogotá: Publicaciones del Instituto Caro y Cuervo.

TANNEN, D. 1996. Género y discurso. Barcelona: Paidós.

THORNBORROW, J. 2012. "Narrative Analysis", en Gee, J. P. y Handford, M. (eds.), The Routledge Handbook of Discourse Analysis. Londres: Routledge, 51-65.

\section{NOTA SOBRE LA AUTORA}

Silvana Guerrero González es doctora en Lingüística y profesora asistente en la Universidad de Chile. 